Check for updates

Cite this: RSC Adv., 2019, 9, 34382

Received 20th August 2019

Accepted 29th September 2019

DOI: $10.1039 / \mathrm{c} 9 \mathrm{ra06540 \textrm {g }}$

rsc.li/rsc-advances

\section{In situ preparation and determination of electrochemical and electrochromic properties of copper phthalocyanine-polyaniline nanocomposite films †}

\author{
Mi Ouyang, ${ }^{a}$ Xuming Hu, ${ }^{a}$ Xiongchao Shao, ${ }^{a}$ Lu Chen, ${ }^{a}$ Weijun $\mathrm{Li}$, (D) $^{\text {a }} \mathrm{Ru}^{\mathrm{Bai}}{ }^{\star \mathrm{b}}$ \\ Ling Zhang, ${ }^{a}$ Xiaojing Lv, ${ }^{a}$ Alexey Tameev ${ }^{c}$ and Cheng Zhang (D) *a
}

Copper phthalocyanine (CuPc) films with different morphologies were electrodeposited on the surfaces of ITO electrodes. Then, in each case, a polyaniline (PANI) film was electrochemically polymerized in situ on the surface of the copper phthalocyanine film to form a CuPc-PANI composite film. The electrochemical properties of the CuPc-PANI composite film were observed to be much better than those of the film without CuPc. With the modification involving the CuPc nanowires, the composite film formed a finer particle surface and an increased interface area between the PANI and the electrolyte. Compared to the single-component PANI film, the CUPc-PANI composite film exhibited better performance with a higher optical contrast $(58 \%$ at $730 \mathrm{~nm}$ ), a faster response speed (coloring time of $1.02 \mathrm{~s}$, discoloring time of $1.96 \mathrm{~s})$, and better cycling stability $(68.71 \%$ of the initial electrochemical activity after 500 cycles, in contrast to only about $48.02 \%$ for PANI). Moreover, the CuPc-PANI film shows a new feature that can be used as a supercapacitor (specifically a capacitance value of about $5.4 \mathrm{mF} \mathrm{cm}^{-2}$ at typical currents). Our results demonstrate that the prepared CUPC-PANI composite film is one of the best candidates for multiple potential applications such as high-performance polymer electrochromic materials and supercapacitors.

\section{Introduction}

Polymer electrochromic (PEC) materials are considered to be the most promising next-generation electrochromic (EC) materials ${ }^{1-5}$ due to their advantages such as easy tailoring of their molecular structure, adjustable energy band, wide range of color change, high optical contrast, fast response, low energy consumption and good processing performance. However, in order to be used in the field of flat displays, a PEC material must meet the requirements of multi-color and even full-color displays, including high cycling stability, fast response speed, etc. At present, the multi-color display properties of PEC materials are mainly achieved through the design and modification of molecular structures, ${ }^{2,6-8}$ such as donor-acceptor (D-A)

\footnotetext{
anternational Sci. \& Tech. Cooperation Base of Energy Material and Application, College of Chemical Engineering, Zhejiang University of Technology, Hangzhou 310014, P. R. China. E-mail: czhang@zjut.edu.cn

${ }^{b}$ Center for Integrated Spintronics, Hangzhou Dianzi University, Hangzhou 310018, $P$. R. China. E-mail: bairu@hdu.edu.cn

${ }^{c}$ The Laboratory for Electronic and Photonic Processes in Polymer Nanocomposites, A. N. Frumkin Institute of Physical Chemistry and Electrochemistry of the Russian Academy of Sciences, Moscow 119071, Russia

$\dagger$ Electronic supplementary information (ESI) available. See DOI: $10.1039 / \mathrm{c} 9 \mathrm{ra} 06540 \mathrm{~g}$
}

structure design, ${ }^{9,10}$ introduction of functional groups, and copolymerization of different monomers. ${ }^{11-13}$ The construction of a cross-linked structural polymer ${ }^{14,15}$ or the combination of a polymer and inorganic material ${ }^{16-18}$ can significantly improve the cycling stability and color change response speed of the PEC materials.

Using organic-inorganic composites has been widely reported to improve the properties of PEC materials. ${ }^{19}$ For example, compared to a PTBTPA film, a PTBTPA/ZnO composite film was reported by Lv et al. ${ }^{20}$ to exhibit a faster switching time and much higher electrochemical stability. Xia et al. ${ }^{21}$ explored $\mathrm{TiO}_{2} / \mathrm{PANI}$ core/shell nanorod array heterostructures as electrochromic materials, and showed them to display four color modes and fast optical switching speeds. Kateb et $a l .{ }^{22}$ prepared a $\mathrm{ZnO} / \mathrm{PEDOT}$ core/shell nanorod array film as an electrochromic electrode; the film showed high contrast, fast switching rate and high coloration efficiency. Despite the remarkable results of these efforts, there are still some problems to be solved, such as interface problems (caused by the difference between polymers and inorganic materials) and the selection of the nanomaterial. The properties of materials can be effectively improved by using nanocomposites, but usually inorganic materials are needed to construct nanostructures, which leads to the interface problem of organic-inorganic materials. In 
contrast, polymer-organic composites can reduce the interface problem and improve the properties of composites to some extent. ${ }^{23}$ However, there have been relatively few reports on polymer-organic composites.

Polyaniline (PANI) and its derivatives constitute a class of typical polymer electrochromic materials. PEC materials with this type of configuration have been widely reported. ${ }^{24-26}$ Although the aniline structure does not necessarily lead to excellent electrochromic properties, the conclusions that come from such versatile structures are more universal and persuasive. Copper phthalocyanine ( $\mathrm{CuPc}$ ) is a class of classical organic photoconductive materials whose electrical conductivity levels differ significantly between light and dark states. ${ }^{27,28}$ It has been reported that $\mathrm{CuPc}$ has been nanocomposited with titanium dioxide to improve its photoconductivity by orders of magnitude..$^{29,30}$

Based on the above considerations, we set out in the work described in this paper to electrodeposit a nano-CuPc film on the surface of an ITO electrode, and in situ composite a polyaniline film by carrying out electrochemical polymerization to obtain a CuPc-PANI nanocomposite film. Such formation of the CuPcPANI nanocomposite was expected to increase the contact interface between polyaniline and the electrode. The introduction of CuPc was expected to improve the interfacial compatibility and electrical conductivity of the composites so as to further enhance the electrochemical properties of the material.

\section{Experimental}

\subsection{Preparation of CuPc nanowires, PANI films and CuPc- PANI composites films}

All solvents and chemicals were of analytical grade and used without further purification. Copper phthalocyanine (CuPc, $>90 \%$ ) and trifluoroacetic acid (TFA, $>99.5 \%$ ) were purchased from Aladdin. The analytical reagents dichloromethane and chloroform were provided by Shanghai Lingfeng Chemical Reagent Co., Ltd. Toluene and acetone were obtained from Hangzhou Shuanglin Chemical Reagent Co., Ltd. Ethanol $(\geq 99.7 \%$ ) was provided by Shanghai Titan Scientific Co., Ltd. Sulfuric acid $\left(\mathrm{H}_{2} \mathrm{SO}_{4}, 95 \%-98 \%\right)$ and sodium hydroxide were purchased from Xilong Chemical Co., Ltd. Aniline (ANI, $\geq 99.5 \%$ ) was obtained from $\mathrm{J} \& \mathrm{~K}$. Transparent indium tin oxide (ITO) glasses (dimensions of $0.9 \mathrm{~cm} \times 4 \mathrm{~cm}$ and sheet resistance $R_{\mathrm{S}} \leq 10 \Omega$ ) were purchased from Guluo Glass Technology Co., Ltd and then ultrasonically washed in distilled water, ethanol, toluene and acetone solutions, respectively.

CuPc nanowires were prepared on the ITO substrates by following a previously reported electrodeposition method. ${ }^{29}$ Copper phthalocyanine at a concentration of $2 \times 10^{-5} \mathrm{M}$ was combined with $40 \mathrm{~mL}$ of chloroform, and then with $1.5 \mathrm{~mL}$ of TFA. A conductive ITO substrate was used as a cathode, and a platinum (Pt) plate was used as an anode. In the copper phthalocyanine solution, electrophoretic deposition was performed at $-8 \mathrm{~V}$, and the deposition amount was $-0.002 \mathrm{C}$. The electrodeposited material was rinsed with methylene chloride and then dried in an oven at $60{ }^{\circ} \mathrm{C}$ for $2 \mathrm{~h}$ and the resulting electrode was labelled as a CuPc electrode.
A PANI thin layer was deposited on the ITO substrate by following the electropolymerization method. The monomer solution was a mixed aqueous solution containing $25 \mathrm{mM}$ aniline and $50 \mathrm{mM}$ sulfuric acid. The electropolymerization was carried out in a three-electrode cell on an ITO substrate as the working electrode, $\mathrm{Ag} / \mathrm{AgCl}$ as the reference electrode, and Pt sheet as the counter electrode. The PANI film was deposited by carrying out bulk electrolysis (BE) with coulometry at a constant potential of $0.8 \mathrm{~V}$ with an electropolymerization charge of $0.06 \mathrm{C}$. (The thickness of the deposited PANI film was about $150 \mathrm{~nm}$.) After electropolymerization, the samples were washed with deionized water and dried in air. And then, the CuPc-PANI film with the above $\mathrm{CuPc}$ nanowires on the ITO substrate was deposited by carrying out BE with coulometry at a constant potential of $0.8 \mathrm{~V}$ with an electropolymerization charge of $0.06 \mathrm{C}$. (The thickness of the deposited CuPc-PANI film was about $200 \mathrm{~nm}$.)

\subsection{Characterization}

The crystal structures and morphologies of the as-prepared samples were characterized by using an X'Pert Pro X-ray diffractometer $(\mathrm{XRD})$ equipped with a $\mathrm{Cu}-\mathrm{K} \alpha$ radiation source $(\lambda=$ $1.5418 \AA$ ), an S-4800 scanning electron microscope (SEM), and a Hitachi HT7700 transmission electron microscope (TEM). Fourier-transform infrared reflection (FTIR) spectra were acquired using a Nicolet 6700 spectrometer (Thermo Fisher Nicolet, USA). The thicknesses of the composite and polymer films were determined by using a DEKTAK-XK step profiler. Cyclic voltammetry (CV), galvanostatic charge/discharge and electrochemical impedance spectroscopy (EIS) were carried out using a CHI660E electrochemical workstation in a three-electrode system containing an aqueous solution of $1 \mathrm{M} \mathrm{H}_{2} \mathrm{SO}_{4}$ as the electrolyte, $\mathrm{Ag} / \mathrm{AgCl}$ as the reference electrode, and a Pt sheet as the counter electrode. Ultraviolet-visible (UV-Vis) absorption spectra were recorded using a UV-1800 UV-Vis spectrometer.

\section{Results and discussion}

FTIR spectra of CuPc, pure PANI, and the CuPc-PANI composite are shown in Fig. 1. Here, it can be seen that CuPc shows intense

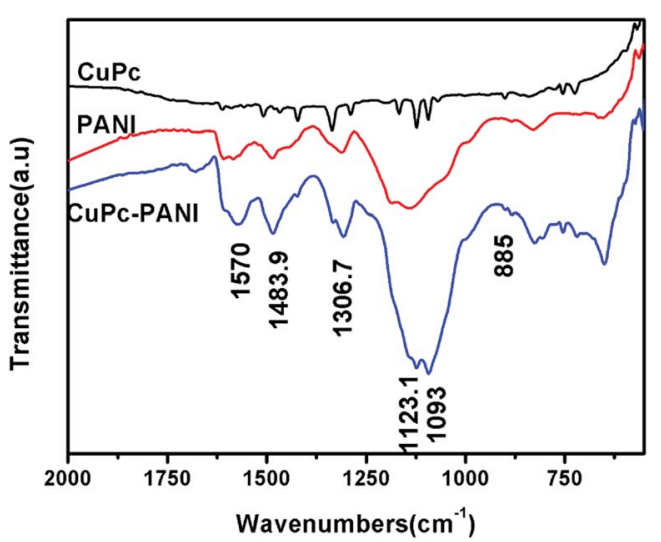

Fig. 1 FTIR spectra of the CuPc film, PANI film, and CUPc-PANI composite film. 
absorption bands at 1612, 1508, 1467, 1422, 1336 and $1289 \mathrm{~cm}^{-1}$, which are derived from the stretching vibration of the planar $\mathrm{C}-\mathrm{C}$ or $\mathrm{C}-\mathrm{N}$ bonds of the phthalocyanine macrocycle. It also yielded bands at around 1167, 1123 and $1094 \mathrm{~cm}^{-1}$, corresponding to the in-plane bending vibration of the benzene ring $\mathrm{C}-\mathrm{H}$ bonds, and bands at 755 and $725 \mathrm{~cm}^{-1}$, attributed to the out-of-plane bending vibration of the benzene ring $\mathrm{C}-\mathrm{H}$ bonds. And the characteristic band at $901 \mathrm{~cm}^{-1}$ caused by the vibration of copper ions and nitrogen atoms on the surrounding four pyrrole rings was also observed; this band is one of the most important features for identifying CuPc. ${ }^{31}$ The presence of these characteristic bands indicates the successful preparation of the CuPc film. The FTIR spectrum of the PANI film is also shown in Fig. 1. Here, the PANI film shows absorption bands at around 1584 and $1487 \mathrm{~cm}^{-1}$, corresponding to the characteristic absorption of the anthracene and benzene rings in the PANI molecular chain. It also yielded a band at $1312 \mathrm{~cm}^{-1}$ from the $\mathrm{C}-\mathrm{N}$ stretching vibration of phenylhydrazine, at around $1141 \mathrm{~cm}^{-1}$ from $\mathrm{C}-\mathrm{N}$ in the benzene ring, and at $829 \mathrm{~cm}^{-1}$ due to the $\mathrm{C}-\mathrm{C}$ bending vibration in the benzene ring. ${ }^{24}$ Fig. 1 also shows the spectrum of CuPc-PANI. This nanocomposite film yielded most of the characteristic bands of PANI and CuPc. For instance, the bands observed at 1484 and $1567 \mathrm{~cm}^{-1}$ are characteristic of the anthracene and benzene rings in the PANI, that at $1307 \mathrm{~cm}^{-1}$ is due to the $\mathrm{C}-\mathrm{N}$ vibration of the PANI, those at 1123 and $1093 \mathrm{~cm}^{-1}$ come from the in-plane bending vibration of the $\mathrm{C}-\mathrm{H}$ bond of the aromatic ring structure of $\mathrm{CuPc}$, that at $1684 \mathrm{~cm}^{-1}$ is caused by the stretching vibrations of $\mathrm{C}=\mathrm{N}$ and $\mathrm{C}=\mathrm{O}$ in the molecular structure of CuPc, and that at $885 \mathrm{~cm}^{-1}$ is caused by the vibration of the copper and nitrogen atoms in the pyrrole ring. The similarity of the three curves indicates that the PANI film was successfully prepared on the CuPc-coated ITO substrate.

The XRD patterns of the CuPc, PANI and CuPc-PANI composite films, each on a conductive ITO substrate, are shown in Fig. 2. After eliminating the intrinsic diffraction peaks of ITO, a sharp diffraction peak appeared at $2 \theta=6.74^{\circ}$ and the corresponding interplanar spacing was measured to be $13.1 \AA$. The intrinsic diffraction peak at $2 \theta=6.64^{\circ}$ confirms that the deposited CuPc film is an $\alpha$-type crystal. The XRD pattern of the CuPc-PANI/ITO composite film is similar to that of the CuPc/

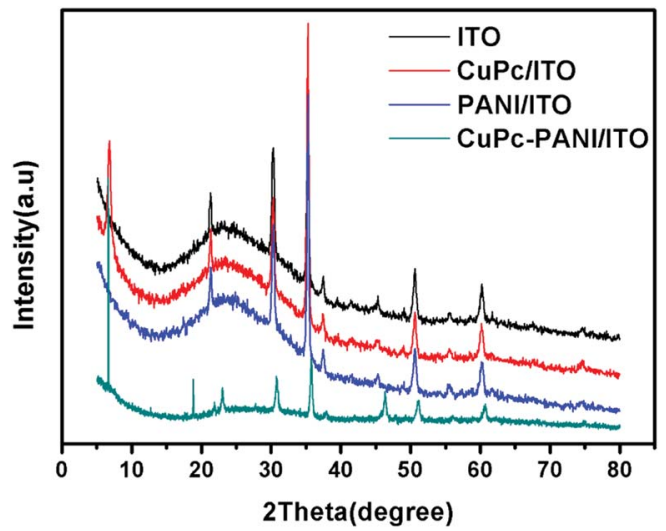

Fig. 2 XRD patterns of ITO glass, CuPc/ITO, PANI/ITO and CuPcPANI/ITO.
ITO film, with this similarity due to the amorphous nature of the PANI film. But the composite film shows new diffraction peaks at $2 \theta=18.84^{\circ}$ and $23.02^{\circ}$. These two peaks derived from the $\beta$-CuPc crystal form, ${ }^{32}$ and their appearance suggests to us that a small amount of $\alpha$-CuPc crystals was converted into $\beta$ CuPc crystals due to a solvation effect, and in this way mixed crystals of CuPc were finally obtained.

The surface morphologies of the CuPc, PANI and CuPc-PANI films were investigated by carrying out SEM and TEM analyses, as shown in Fig. 3. The ITO substrates prepared with CuPc and PANI are observed to be covered with a layer of CuPc nanowires (Fig. 3a and c) and a layer of PANI nanoparticles (Fig. S1 $\dagger$ ), respectively. The CuPc nanowires show lengths of 1-2 $\mu \mathrm{m}$ and diameters of about 20-100 $\mathrm{nm}$. Moreover, the morphology and distribution of the CuPc nanowires are observed to be dendritic. Smaller, shorter nanowires appear to have gradually coalesced to form thicker, longer micron-sized lines. The average diameters of the PANI nanoparticles range from 20 to $60 \mathrm{~nm}$. Fig. $3 \mathrm{~b}$ and d show a layer of PANI nanoparticles grown on a CuPc substrate. Inspection of these images indicates that the PANI particles remained evenly distributed after being compounded with CuPc. The outline of the CuPc nanowires under the PANI membrane can be roughly seen from the surface of the composite film.

This observation demonstrates that the CuPc-PANI composite film was formed in situ. TEM was also used to analyze the structural characteristics of the CuPc nanowires and CuPc-PANI composite film. Here, as shown in Fig. 3e, the thin $\mathrm{CuPc}$ nanowires are observed to be aggregated together, and their morphologies and dimensions are consistent with the results of the corresponding SEM images. After electrochemical
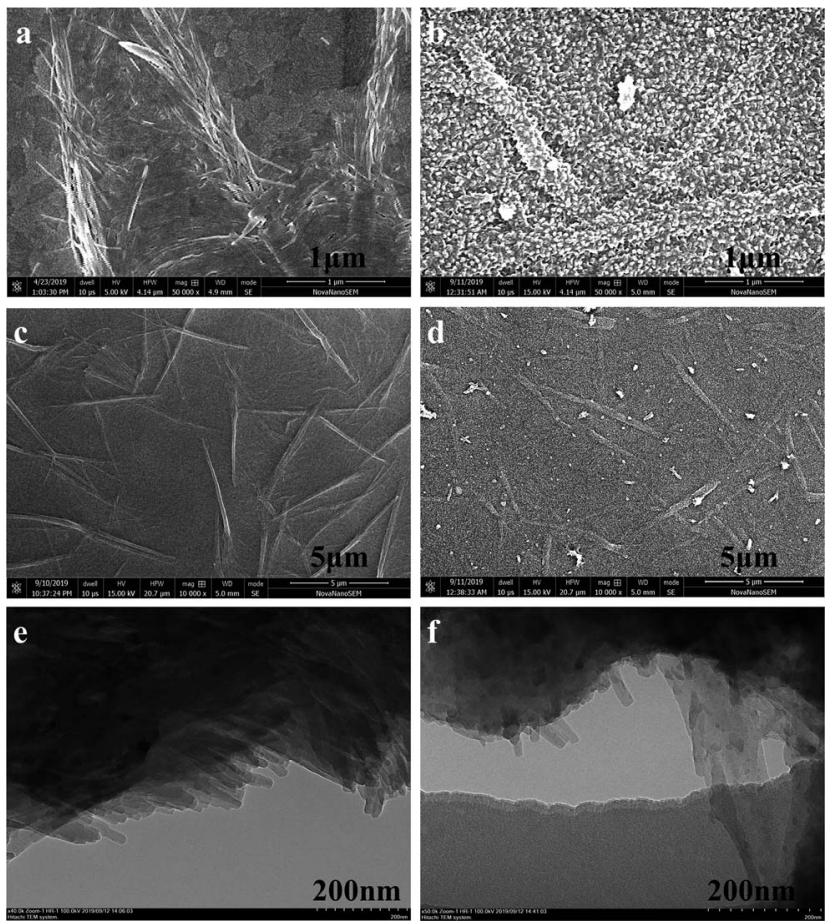

Fig. 3 ( $a-d)$ SEM images of ( $a$ and $c$ ) CuPc and ( $b$ and d) the CuPcPANI composite film at various magnifications. (e and f) TEM images of (e) CuPc and (f) the CuPc-PANI composite film. 
polymerization of PANI, the presence of CuPc nanowires in CuPc-PANI composites was also observed, as shown in the TEM image (Fig. 3f) of a small piece of thin film peeling off during the ultrasonic preparation of the composite sample. Several nanowires can be clearly seen jutting out from the thin film. The morphology and dimensions of these nanowires are consistent with the morphology and dimensions of the CuPc nanowires. This observation also confirms the successful preparation of the CuPc-PANI composite film.

Fig. 4 shows the UV-Visible absorption spectra of the PANI and CuPc-PANI composite films acquired under various applied voltages ranging from -0.2 to $0.7 \mathrm{~V}$. As the applied voltage was increased from -0.2 to $0.2 \mathrm{~V}$, the absorption peak at $410 \mathrm{~nm}$ increased, but then decreased as the voltage was further increased from 0.2 to $0.7 \mathrm{~V}$. This result can be explained by the formation of a bipolar species at higher voltage, which has been shown to lead to a reduction in the population of polarons. ${ }^{24}$ In the -0.2 to $0.7 \mathrm{~V}$ range, the characteristic absorbance band close to $730 \mathrm{~nm}$ and related to the $\pi-\pi^{*}$ transition in the quinoid ring of PANI can be observed. At low voltages $(-0.2$ to $0 \mathrm{~V}$ ), an absorption band was also be observed at about $860 \mathrm{~nm}$, which made the PANI film appear pale yellow and the CuPcPANI composite film pale blue (related to the light blue appearance of the CuPc film) when the PANI and CuPc-PANI films were in the reduced state. It is worth noting that changing the voltage produced similar changes in the UV-Vis absorption spectra of the CuPc-PANI composite film and PANI film, indicating that combining CuPc with PANI did not significantly change the UV absorption properties of PANI. Therefore, the composite film still retained the original electrochromic properties of PANI.

Fig. 5a shows the recorded CV curves of PANI, CuPc and CuPc-PANI films in an aqueous solution of $1 \mathrm{M} \mathrm{H}_{2} \mathrm{SO}_{4}$ from -0.2 to $0.7 \mathrm{~V}$ at a scanning rate of $100 \mathrm{mV} \mathrm{s}^{-1}$. The $\mathrm{CV}$ curves of the PANI and CuPc-PANI films were observed to substantially coincide, each showing an oxidation peak at $0.3 \mathrm{~V}$ and reduction peak at about $0.0 \mathrm{~V}$. Moreover, the CuPc-PANI composite film was concluded to have retained good electrochemical activity. The inset depicts an enlarged view of the CV curve for the CuPc film. Inspection of this curve showed two weak oxidation peaks, at 0.5 and $0.67 \mathrm{~V}$, and the corresponding reduction peaks at 0.5 and $0.69 \mathrm{~V}$, with these results due to the weak electrochromic properties of CuPc, i.e., it not changing color between -0.2 and 0.7 V. In contrast, the PANI film in the CV test showed a color
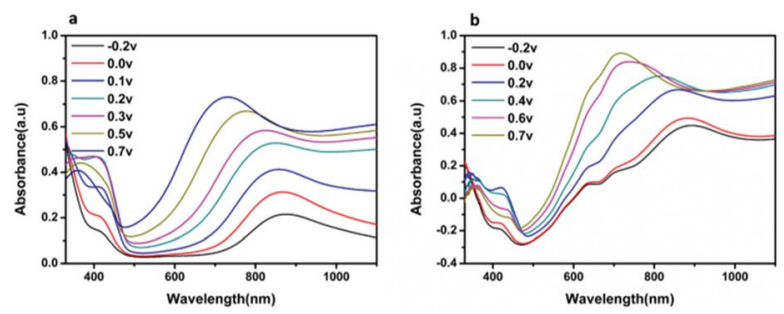

Fig. 4 UV-Vis absorption spectra of (a) pure PANI and (b) the CuPcPANI composite film, each acquired at various voltages ( -0.2 to $0.7 \mathrm{~V}$ ) in a $1 \mathrm{M} \mathrm{H}_{2} \mathrm{SO}_{4}$ solution.

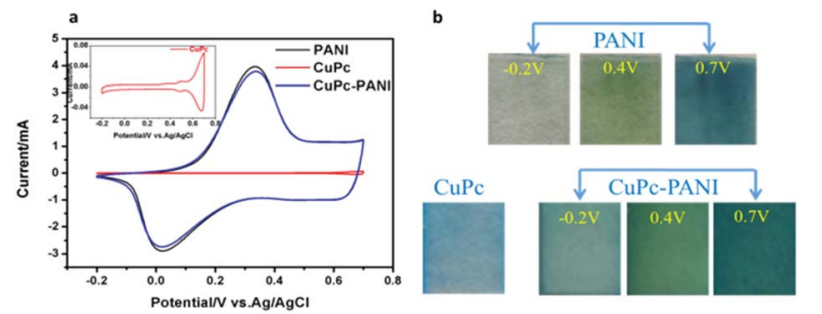

Fig. 5 (a) Cyclic voltammetry curves of pure PANI and CuPc-PANI composite films recorded in a $1 \mathrm{M} \mathrm{H}_{2} \mathrm{SO}_{4}$ aqueous solution at a scan rate of $100 \mathrm{mV} \mathrm{s}^{-1}$. (b) Photographic images of the CuPc film and of the pure PANI and CUPC-PANI films at various applied voltages.

change from pale yellow to yellowish green to blue as the voltage was increased from $-0.2 \mathrm{~V}$ to $0.7 \mathrm{~V}$. Photographic images of PANI and CuPc-PANI films in discolored $(-0.2 \mathrm{~V})$ and colored $(0.7 \mathrm{~V})$ states are shown in Fig. $5 \mathrm{~b}$. At $-0.2 \mathrm{~V}$, the PANI was reduced and appeared transparent, while the CuPc-PANI film appeared as weak blue. This result can be mainly attributed to the light blue of the CuPc film at this voltage. When the applied voltage was increased to $0.7 \mathrm{~V}$, both films showed blue color. As shown in Fig. 5b, the uniformity of the CuPc-PANI film is obviously similar to that of the PANI film, indicating that the introduction of the CuPc nanostructure did not have much of a negative effect on the formation of the PANI film.

Electrochemical stability levels of the pure PANI film and CuPc-PANI nanocomposite film were determined by carrying out CV scanning for 500 cycles between $-0.2 \mathrm{~V}$ and $0.7 \mathrm{~V}$ in a $1 \mathrm{M} \mathrm{H}_{2} \mathrm{SO}_{4}$ aqueous solution (as shown in Fig. 6). After 500 cycles, the CV curves of both the PANI film and CuPc-PANI nanocomposite film showed decreases in electrochemical stability. The electroactivity of the PANI film decreased by $51.98 \%$, while that of the CuPc-PANI nanocomposite film decreased by only $31.29 \%$. The result suggested that the electrochemical stability of the composite film was obviously enhanced as a result of the introduction of the CuPc nanowires. In general, the performance degradation of a conductive polymer is inevitable in the process of repeated doping/dedoping. Poor adhesion of the conductive polymer to the substrate is an important cause of low electrode performance in long-term operation. The conductive ITO substrate is an inorganic material with a smooth surface and the conductive polymer is an organic material. There are some differences in the properties
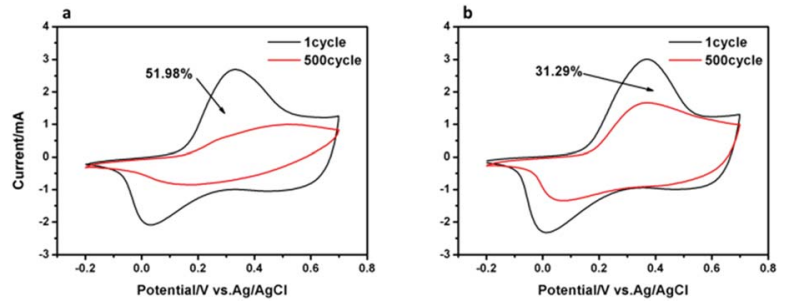

Fig. 6 The 1st cycle (dark line) and 500th cycle (red line) CV curves of (a) the pure PANI film and (b) CuPc-PANI composite film as a function of repeated scans in $1 \mathrm{M} \mathrm{H}_{2} \mathrm{SO}_{4}$ between -0.2 and $0.7 \mathrm{~V}$. Scan rate: $100 \mathrm{mV} \mathrm{s}^{-1}$. 
of the two materials, such as intermolecular and intramolecular forces, i.e., physical and chemical properties. Therefore, the interfacial adhesion between the two compositions is not very good. ${ }^{33}$ In the case of repeated doping and dedoping, the substrate-polymer joint is gradually corroded, which leads to a worsening of electrochromic properties. Herein, the introduction of CuPc nanostructures between the PANI film and the ITO substrate provided multi-angle and non-planar interfacial contacts and significantly increased the effective area between the PANI and CuPc-coated electrodes. Thus, such a composite structure with enhanced interfacial adhesion can increase the long-term electrochemical stability of electrochromic materials and devices. $^{34}$

The electrochromic switching behaviours of the PANI film and CuPc-PANI composite film were monitored at the wavelength of maximum absorption in order to characterize their optical contrasts and response times. Fig. 7 displays the optical contrast curves of the PANI film and CuPc-PANI composite film at $730 \mathrm{~nm}$ in an aqueous solution of $1 \mathrm{M} \mathrm{H}_{2} \mathrm{SO}_{4}$ between $-0.2 \mathrm{~V}$ and $0.7 \mathrm{~V}$ with a residence time of $10 \mathrm{~s}$. The optical contrast of the PANI film was $56 \%$, and that of the CuPc-PANI film was $58 \%$. The introduction of CuPc nanowires did not significantly improve the optical contrast of PANI. When PANI was bleached to pale yellow, the color of the composite film remained light blue due to the overlay of the CuPc layer.

Response time is an important measure of the performance of an electrochromic material and it is defined as the time it takes for a material to reach $95 \%$ of its full change in absorbance after switching of the voltage. Fig. 8 shows the response time of the PANI film and CuPc-PANI composite film at $730 \mathrm{~nm}$. The PANI film displayed a discoloring time of $2.9 \mathrm{~s}$ and a coloring time of $0.9 \mathrm{~s}$. For comparison, the CuPc-PANI displayed a discoloring time of $1.96 \mathrm{~s}$ and a coloring time of $1.02 \mathrm{~s}$. In other words, the results showed a shorter discoloring time for the CuPc-PANI nanocomposite film than for the PANI film. This difference was speculated to have been due to the introduction of CuPc nanowires having improved the surface morphology of the PANI film, specifically having increased the contact area between PANI and electrolyte solution, which would have facilitated the doping/dedoping of ions and their diffusion in the polymer film, thus improving the response time. The electrochromic performance of the CuPc-PANI composite film in the current work was also better than that of PANI-base composite films reported in some of the literature (Table $\mathrm{S} 1 \dagger$ ).
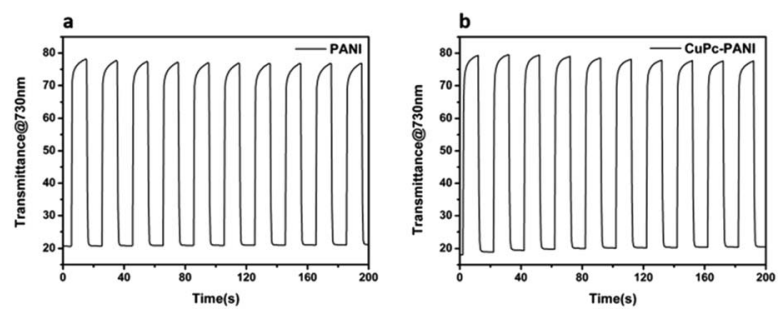

Fig. 7 Kinetic optical transmittance curves at $730 \mathrm{~nm}$ of (a) pure PANI and (b) the CuPc-PANI composite film, each subjected to switching between -0.2 and $0.7 \mathrm{~V}$ for $10 \mathrm{~s}$ at each step in an aqueous solution of $1 \mathrm{M} \mathrm{H}_{2} \mathrm{SO}_{4}$.
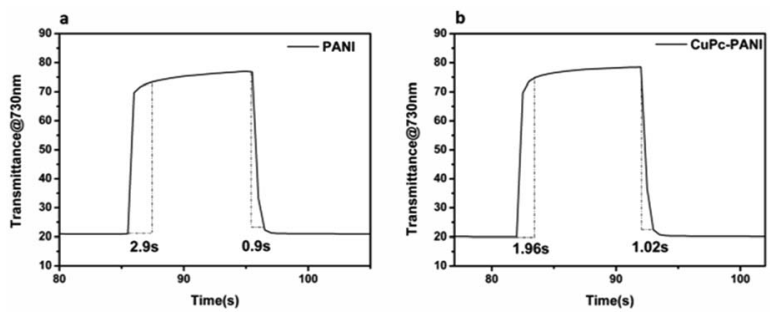

Fig. 8 Response times at $730 \mathrm{~nm}$ of (a) pure PANI and (b) the CuPcPANI composite film

In addition, considering that the thickness and areal dimensions of the CuPc film has been shown to change with a change of electrodeposition charge, the effect of the magnitude of the electric charge of CuPc on the electrochromic properties of the composite film were studied (as shown in Table S2 and Fig S2 $\dagger$ ). The optimized data were used for the experiments described in this paper.

Fig. 9 shows a possible mechanism for the diffusion of the counterions in the PANI film and the CuPc-PANI composite film. According to this proposed mechanism, when a voltage is applied, the counterions in the electrolyte would enter the PANI film and then diffuse using different paths in the film. The slow response speed of the PANI film was attributed to the long diffusion distance and slow diffusion of the counterions in the film. The introduction of CuPc nanowires in the PANI film was modeled to improve the diffusion of counterions in the PANIs. An application of voltage would cause the surface of CuPc on the ITO substrate to become positively charged. Entry of the counterions of the electrolyte into the PANI film would result in a shortening of the diffusion distance, and an increase in the density of available contact sites, which would in turn speed up the diffusion of counterions and improve the response speed of the PANI film.

Nyquist plots are often used to investigate the charge transfer impedance and ion diffusion process of electrochromic materials. The Nyquist plots of the two films (Fig. 10) each show a semicircle in the high-frequency region and an inclined line in the low-frequency region. The high-frequency semicircle

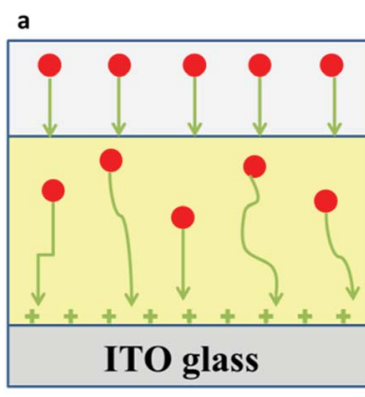

counterion

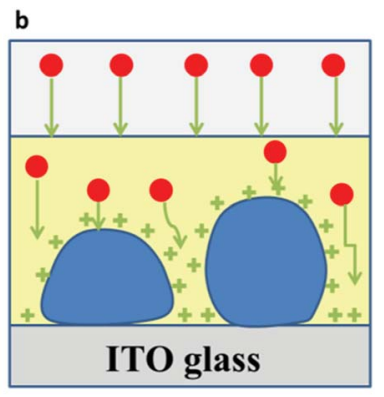

electrolyte solution

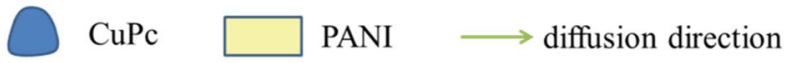

Fig. 9 The behaviors of counterion transport in (a) the PANI film and (b) CuPc-PANI composite film. 


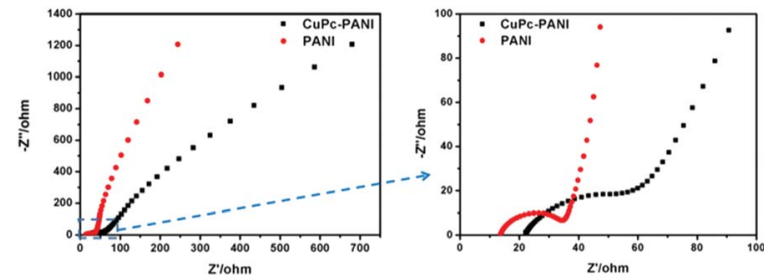

Fig. 10 Nyquist plots of PANI and the CuPc-PANI composite film. EIS measurements were conducted in the frequency range $0.1 \mathrm{~Hz}$ to 100 $\mathrm{kHz}$ in an aqueous solution of $1 \mathrm{M} \mathrm{H}_{2} \mathrm{SO}_{4}$. The right panel shows a larger version of the blue box of the left panel.

corresponded to the charge transfer impedance at the electrode/ electrolyte interface, and the inclined line in the low-frequency region was assigned to the ion diffusion process within the electrode. It is well known that a larger semicircle means a higher charge transfer resistance, and a steeper slope means a lower ion diffusion rate..$^{19}$ Our results demonstrate faster ion diffusion and greater charge transfer resistance for the CuPcPANI composite film than for the PANI film. As an organic semiconductor material with photoconductivity properties, the conductivity of CuPc was observed to be not as high as that of ITO, which led to the large charge transfer impedance of the composite film. However, the introduction of the CuPc nanostructure increased the contact area between polyaniline and electrolyte, which was conducive to the doping/dedoping of ions and ion transport, and improved the rate of diffusion of the ions.

Interestingly, in addition to the excellent electrochromic properties of the CuPc-PANI composite film, we also found it displaying supercapacitor properties. The CV curves of the CuPc-PANI composite film acquired at different scan rates (Fig. S3†) exhibited each a nearly rectangular shape, which demonstrated that the composite film displayed pseudocapacitance characteristics. ${ }^{35,36}$ The supercapacitive performance was investigated by evaluating the charge-discharge behaviour of the CuPc-PANI composite film in an aqueous solution of $1 \mathrm{M} \mathrm{H}_{2} \mathrm{SO}_{4}$ at a series of applied currents using a voltage window from -0.2 to $0.7 \mathrm{~V}$. The area of the CuPcPANI composite film that was measured was $2.34 \mathrm{~cm}^{2}$. The areal capacitance $\left(\mathrm{C}, \mathrm{mF} \mathrm{cm}^{-2}\right)$ of the CuPc-PANI composite film was calculated from discharge curves using the equation $C=(I \Delta t / S \Delta V)$, where $I$ is the discharge current, $\Delta t$ is the discharge time, $\Delta V$ is the potential window, and $S$ is the effective area of electrode. As shown in Fig. 11a, areal capacitances of the CuPc-PANI composite film were measured at various currents to be in the vicinity of $\sim 5.4 \mathrm{mF}$ $\mathrm{cm}^{-2}$ This capacitance value was smaller than that of the $\mathrm{W}_{18} \mathrm{O}_{49} /$ PANI composite reported by the Zhao group $(10 \mathrm{mF}$ $\left.\mathrm{cm}^{-2}\right) \cdot{ }^{37}$ The vertical array of $\mathrm{W}_{18} \mathrm{O}_{49}$ nanowires and the thick PANI film may have been the main reasons for the better capacitance performance. The charge-discharge curves of the CuPc-PANI composite showed a non-ideal triangular shape due to the redox process, indicating that the composite film displayed the typical pseudocapacitance characteristics and fast charge-discharge ability. ${ }^{37}$ Fig. 11b
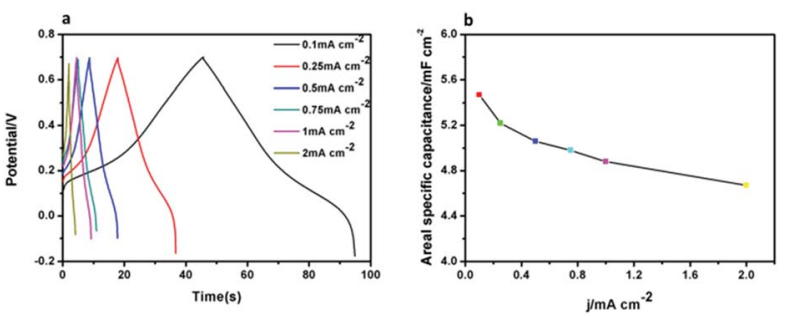

Fig. 11 (a) Galvanostatic charge-discharge curves of the CuPc-PANI composite film. (b) Areal specific capacitance of the CuPc-PANI composite film as a function of current density.

shows the areal specific capacitance values of the CuPc-PANI composite film at various current densities. It is common for the areal capacitance to decrease with an increase in current density. This phenomenon can explain that at a higher current density, the slow transfer of doping ions produces dynamic resistance, which affects the charge transfer process on the electrode/electrolyte interface, so that some polymer chains cannot fully participate in the redox reaction. ${ }^{37,38}$

\section{Conclusions}

A novel CuPc-PANI composite was prepared in situ by following the electrochemical method. The composite film showed better electrochromic properties such as higher optical contrast, faster response speed and better cycle stability, than did the PANI film. The improvement of electrochromic performance was mainly attributed to the introduction of CuPc nanostructures having increased the contact area between the conductive polymer and the electrolyte, in turn resulting in facilitated ion diffusion and increased surface area for charge transfer. Moreover, the composite film also showed obvious supercapacitor properties, specifically showing an areal capacitance of $\sim 5.4 \mathrm{mF} \mathrm{cm}{ }^{-2}$ at various currents. This result suggested that the composites could be used not only in the electrochromic field, but also in energy storage.

\section{Conflicts of interest}

There are no conflicts to declare.

\section{Acknowledgements}

The authors are grateful for support from the National Natural Science Foundation of China (51573165, 51673174, 51603185, 51703199) and the Natural Science Foundation of Zhejiang Province (LZ17E030001, LY19E030006, LQ17F010004). They are especially thankful for the discussion of pseudocapacitance data with Prof. Chang Su of the College of Chemical Engineering at Shenyang University of Chemical Technology, and the collection of TEM data by Qian Yang of the Research Center of Analysis and Measurement at Zhejiang University of Technology. 


\section{References}

1 A. A. Argun, P. H. Aubert, B. C. Tompson, I. Schwendeman, C. L. Gaupp, J. Hwang, N. J. Pinto, D. B. Tanner, A. G. MacDiarmid and J. R. Reynolds, Chem. Mater., 2004, 16(23), 4401-4412.

2 M. Sezgin, O. Ozay, S. Koyuncu, H. Ozay and F. Baycan Koyuncu, Chem. Eng. J., 2015, 274, 282-289.

3 S. L. Ming, S. J. Zhen, K. W. Lin, L. Zhao, J. K. Xu and B. Y. Lu, ACS Appl. Mater. Interfaces, 2015, 7(21), 11089-11098.

4 B. Y. Lu, S. J. Zhen, S. M. Zhang, J. K. Xu and G. Q. Zhao, Polym. Chem., 2014, 5(17), 4896-4908.

5 J. Jensen, M. Hosel, A. L. Dyer and F. C. Krebs, Adv. Funct. Mater., 2015, 25(14), 2073-2090.

6 K. Wang, T. Zhang, Y. H. Hu, W. G. Yang and Y. Shi, Electrochim. Acta, 2014, 130, 46-51.

7 G. Atakan and G. Gunbas, RSC Adv., 2016, 6(30), 2562025623.

8 S. Zhao, W. D. Huang, Z. S. Guan, B. Jin and D. B. Xiao, Electrochim. Acta, 2019, 298, 533-540.

9 B. Hu, X. J. Lv, J. W. Sun, G. F. Bian, M. Ouyang, Z. Y. Fu, P. J. Wang and C. Zhang, Org. Electron., 2013, 14(6), 15211530.

10 P. M. Beaujuge, S. Ellinger and J. R. Reynolds, Nat. Mater., 2008, 7(10), 795-799.

11 A. J. C. Silva, S. M. F. Ferreira, D. D. Santos, M. Navarro, J. Tonholo and A. S. Ribeiro, Sol. Energy Mater. Sol. Cells, 2012, 103, 108-113.

12 L. L. Ji, Y. Y. Dai, S. M. Yan, X. J. Lv, C. Su, L. H. Xu, Y. K. Lv, M. Ouyang, Z. F. Chen and C. Zhang, Sci. Rep., 2016, 6, 0068.

13 Y. J. Tao, K. Zhang, Z. Y. Zhang, H. F. Cheng, C. L. Jiao and Y. L. Zhao, Chem. Eng. J., 2016, 293, 34-43.

14 Y. Y. Dai, W. J. Li, X. X. Qu, J. Liu, S. M. Yan, M. Ouyang, X. J. Lv and C. Zhang, Electrochim. Acta, 2017, 229, 271-280.

15 Y. J. Dong, F. F. Luo, L. Chen, F. Y. Yuan, Y. J. Hou, W. J. Li, S. M. Yan, Y. Y. Dai, M. Ouyang and C. Zhang, Phys. Chem. Chem. Phys., 2018, 20, 12923-12928.

16 J. L. Lu, W. S. Liu, H. Ling, J. H. Kong, G. Q. Ding, D. Zhou and X. H. Lu, RSC Adv., 2012, 2, 10537-10543.

17 J. H. Zhu, S. Y. Wei, M. Alexander, T. D. Dang, T. C. Ho and Z. H. Guo, Adv. Funct. Mater., 2010, 20(18), 3076-3084.

18 Z. Q. Tong, S. K. Liu, X. G. Li, J. P. Zhao and Y. Li, Nanoscale Horiz., 2018, 3, 261-292.

19 G. F. Cai, J. P. Tu, D. Zhou, J. H. Zhang, Q. Q. Xiong, X. Y. Zhao, X. L. Wang and C. D. Gu, J. Phys. Chem. C, 2013, 117, 15967-15975.
20 X. J. Lv, J. W. Sun, B. Hu, M. Ouyang, Z. Y. Fu, P. J. Wang, G. F. Bian and C. Zhang, Nanotechnology, 2013, 24(26), 265705.

21 X. H. Xia, D. L. Chao, X. Y. Qi, Q. Q. Xiong, Y. Q. Zhang, J. P. Tu, H. Zhang and H. J. Fan, Nano Lett., 2013, 13(9), 4562-4568.

22 M. Kateb, S. Safarian, M. Kolahdouz, M. Fathipour and V. Ahamdi, Sol. Energy Mater. Sol. Cells, 2016, 145, 200-205.

23 H. Randriamahazaka, V. Noel, S. Guillerez and C. Cgevrot, J. Electroanal. Chem., 2005, 585(2), 157-166.

24 S. H. Zhang, G. Sun, Y. F. He, R. F. Fu, Y. C. Gu and S. Chen, ACS Appl. Mater. Interfaces, 2017, 9, 16426-16434.

25 F. Hu, J. L. Xu, S. H. Zhang, J. Jiang, B. Yan, Y. C. Gu, M. J. Jiang, S. J. Lin and S. Chen, J. Mater. Chem. C, 2018, 6(21), 5707-5715.

26 C. L. Lin and L. J. Liao, Sol. Energy Mater. Sol. Cells, 2015, 145, 54-60.

27 F. M. Liu, J. Sun, S. Xiao, W. L. Huang, S. H. Tao, Y. Zhang, Y. L. Gao and J. L. Yang, Nanotechnology, 2015, 26(22), 225601.

28 S. Nagel, M. Lener, C. Keil, R. Gerdes, L. Lapok, S. M. Gorun and D. Schlettwein, J. Phys. Chem. C, 2011, 115(17), 87598767.

29 M. Ouyang, R. Bai, L. Chen, L. G. Yang, M. Wang and H. Z. Chen, J. Phys. Chem. C, 2008, 112(30), 11250-11256.

30 M. Ouyang, R. Bai, L. G. Yang, Q. Chen, Y. G. Han, M. Wang, Y. Yang and H. Z. Chen, J. Phys. Chem. C, 2008, 112(7), 23432348.

31 Z. C. Wang, R. B. Wei and X. B. Liu, J. Mater. Sci., 2016, 51, 4682-4690.

32 B. Li, C. Bao, B. X. Shi, K. Tomonori and H. Mitsuo, J. Infrared, Millimeter, Terahertz Waves, 2002, 21(4), 257-260.

33 L. Zhao, L. Zhao, Y. X. Xu, T. F. Qiu, L. J. Zhi and G. Q. Shi, Electrochim. Acta, 2009, 55(2), 491-497.

34 X. J. Lv, B. Hu, J. W. Sun, M. Ouyang, C. H. Yu, Z. Y. Fu and C. Zhang, J. Electrochem. Soc., 2013, 160(2), H87-H92.

35 Y. Zhong, Z. S. Chai, Z. M. Liang, P. Sun, W. G. Xie, C. X. Zhao and W. J. Mai, ACS Appl. Mater. Interfaces, 2017, 9(39), 3408534092 .

36 K. J. Sun, H. Peng, J. J. Mu, G. F. Ma, G. H. Zhao and Z. Q. Lei, Ionics, 2015, 21(8), 2309-2317.

37 Y. Y. Tian, S. Cong, W. M. Su, H. Y. Chen, Q. W. Li, F. X. Geng and Z. G. Zhao, Nano Lett., 2014, 14(4), 2150-2156.

38 Q. F. Guo, J. J. Li, B. Zhang, G. M. Nie and D. B. Wang, ACS Appl. Mater. Interfaces, 2019, 11, 6491-6501. 